Musées, Patrimoine et Culture scientifiques et techniques

$170 \mid 2017$

mars-avril 2017

\title{
La représentation des Allemands dans les musées du Débarquement en Normandie : entre histoire et mémoire
}

Constance Micalef Margain

\section{OpenEdition}

Journals

Édition électronique

URL : http://journals.openedition.org/ocim/1755

DOl : 10.4000/ocim. 1755

ISSN : 2108-646X

Éditeur

OCIM

Édition imprimée

Date de publication : 1 mars 2017

Pagination : 23-27

ISSN : 0994-1908

\section{Référence électronique}

Constance Micalef Margain, « La représentation des Allemands dans les musées du Débarquement en Normandie : entre histoire et mémoire », La Lettre de l'OCIM [En ligne], 170 | 2017, mis en ligne le 01 mars 2018, consulté le 19 avril 2019. URL : http://journals.openedition.org/ocim/1755 ; DOI :

10.4000/ocim. 1755

Ce document a été généré automatiquement le 19 avril 2019

Tous droits réservés 


\section{La représentation des Allemands dans les musées du Débarquement en Normandie : entre histoire et mémoire}

Constance Micalef Margain

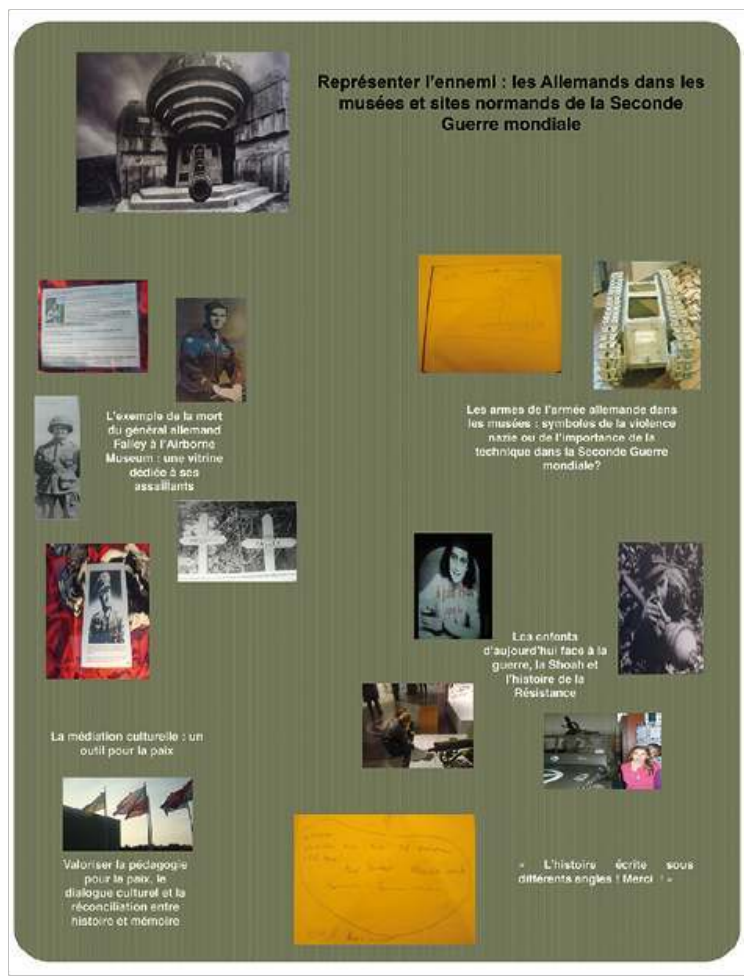

(c) Constance Micalef-Margain 
1 La Normandie a payé plus qu'aucune autre région, un prix élevé en vies humaines et en destructions lors du Débarquement et de la Bataille de Normandie qui se sont déroulés entre le 6 juin et le 29 août 1944. Dix ans furent nécessaires à sa reconstruction. La "mémoire du silence", les tombes militaires ou civiles, les plages du Débarquement aux nombreuses signalétiques, les espaces muséaux ou encore les restes du Mur de l'Atlantique interrogent les relations entre histoire et mémoire. L'espace est criblé de musées locaux (le musée du Débarquement d'Arromanches, l'Airborne Museum...) aux exigences scientifiques, aux moyens financiers, aux statuts (associatifs, privés) ou aux surfaces d'exposition, divers.

2 Un seul aspect dans le panel non exhaustif de musées visités les unit: la représentation de l'ennemi, l'Allemand. Cette représentation reflète une mémoire fanée de l'événement pour ne pas dire passéiste. L'adversaire allemand est finalement peu ou mal représenté. Si nuance il y a, notre propos est d'illustrer le difficile passage du tourisme de souvenir (le temps des commémorations officielles, des héros-vainqueurs) au tourisme d'histoire dans quelques-uns de ces espaces muséaux.

L'Airborne Museum expose des tombes allemandes comme des trophées de guerre.

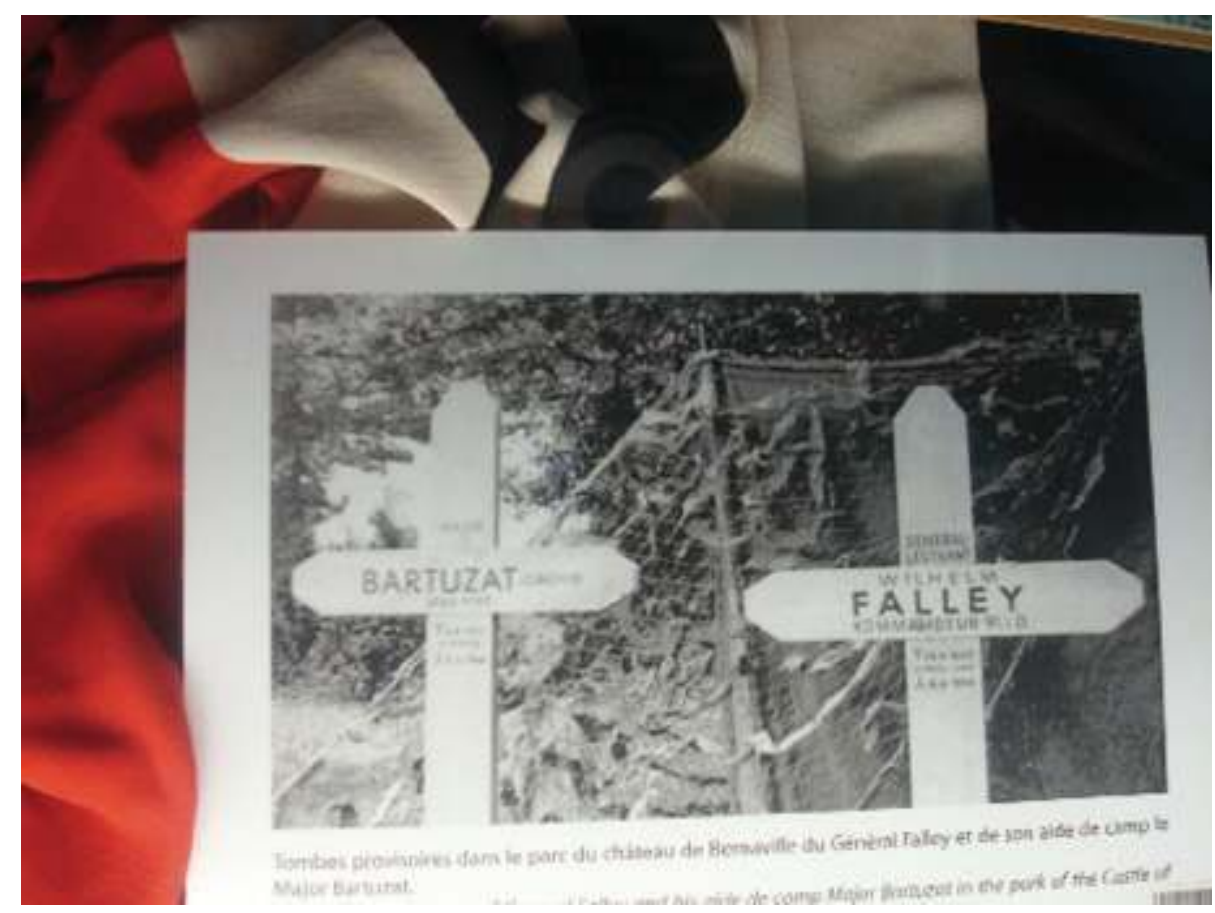

(c) Constance Micalef-Margain

3 En juin 2015 un ami allemand qui visitait ces musées, se disait étonné de la liesse qui entourait les commémorations du 6 juin en Normandie. Il aurait préféré que cette victoire soit célébrée dans une certaine "dignité". Cette dissension interroge. Il s'agit de l'analyser à la lumière de la documentation muséale. L'enjeu est de taille : le dialogue francoallemand des mémoires face à la mise en scène de "passés douloureux" dans les musées et sites français, n'interroge pas moins que la construction de l'espace pacifié européen. 


\section{Les crimes de la Wehrmacht et des nazis dans les musées du Débarquement}

4 L'exemple de la représentation de la mort du général allemand Wilhelm Falley (1897-1944) à l'Airborne Museum ${ }^{1}$ illustre la difficulté de représenter l'ennemi sur un territoire profondément marqué par la guerre. Au sein de l'espace "Waco", une vitrine présente des photographies de soldats américains, présentés comme des héros, à l'origine de la mort du général allemand Wilhelm Falley et du lieutenant-colonel Joachim Bartuzat (1897-1944). Alors que ces derniers se rendaient à Rennes, Falley demanda alors à son chauffeur de rebrousser chemin pour retourner à son quartier général logé dans le château de Bernaville, près de Sainte-Mère-Église. Son véhicule tomba dans une embuscade tendue par des parachutistes américains le 6 juin 1944 au matin. Survivant de la fusillade, il fut tué par un soldat américain alors qu'il tentait récupérer son arme. Ce dernier ne savait pas au moment des faits, qu'il avait tiré sur un officier de haut rang. Dans cette même vitrine, on peut voir la photographie du général (vivant) posée sur un drapeau aux insignes pris par les soldats dans sa voiture. Enfin, une autre photographie montre sa sépulture et celle de l'officier qui l'accompagnait.

5 Sans évoquer l'article 13 des Conventions de Genève sur les prisonniers de guerre qui spécifie que les prisonniers doivent être traités avec humanité et qu'il est interdit de les tuer, cette représentation de la mort du général Falley communique de fait un message à la gloire de l'armée américaine mais sans contextualisation historique. En effet, la mort de ce général est présentée comme un trophée alors qu'il serait possible de chercher à comprendre comment, par son récit, cette mort provoqua au bénéfice des Alliés, des conséquences sur le déroulement du Débarquement et retarda de fait la contre-offensive allemande. De même, dans un souci de clarification les biographies de l'ensemble des protagonistes allemands seraient un apport historique non négligeable, en quelques courts cartels. Nous pourrions rajouter rétrospectivement, les sentiments des soldats américains. En effet, puisque l'attaque a été brutale et non préméditée, ils ne pouvaient avoir conscience au moment des faits, de l'importance de ce trophée, en l'occurrence d'avoir tué un général nazi. Exposer, c'est mettre les objets au service d'un discours ${ }^{2}$. Si ce discours est incomplet et propose une interprétation discutable, comment le public peutil s'approprier cette histoire pour construire sa propre narration?

6 Que penser par ailleurs, dans l'espace "C-47" dans une vitrine en hauteur de ce même musée, de l'assemblage de drapeaux nazis, d'armes, d'un pyjama rayé (symbole de l'univers concentrationnaire), de couverts (symbole de la vie quotidienne des soldats) et d'objets appartenant à des soldats ? Ce pêle-mêle scénographique soulève un problème historiographique majeur : le degré de compromission de l'armée allemande avec les crimes nazis.

7 Cette problématique n'est pas résolue dans le musée d'Utah Beach ${ }^{3}$ malgré une présentation très précise des militaires allemands dans les premières salles du musée. Dans une de ces salles, la première, nous trouvons un cartel sous des chaussures portées par des soldats allemands où il est indiqué : "D'un poids de $1,5 \mathrm{~kg}$ chacune, ces bottes chaudes mais encombrantes équipaient certaines catégories de soldats comme les sentinelles sur le front russe. Leur feutrine était composée de cheveux humains prélevés sur les Juifs assassinés par millions dans les camps d'extermination". La relativité du chiffre de personnes juives assassinées pose question. S'il est certain que les cheveux des 
Juifs assassinés étaient récupérés par la machine industrielle nazie, là aussi, l'absence de contextualisation historique, mène à des confusions sémiologiques.

Un autre exemple de l'utilisation de l'histoire de la Shoah à des fins spectaculaires, est la reproduction photographique d'Anne Frank marquée du 6 juin 1944 et qui se trouve au fond de l'ancien bunker du général allemand Friedrich-Wilhelm Richter (1911-1989) réaménagé en espace muséal au Mémorial de Caen. Anne Frank, Allemande d'origine juive est née en 1929 et morte entre février et mars 1945 dans le camp de concentration de Bergen-Belsen. Le 6 juin 1944, Anne Frank est encore cachée à Amsterdam avec sa famille. Elle est arrêtée le 4 août 1944. La publication du journal d'Anne Frank a fait du destin de cette jeune fille, un symbole de la barbarie nazie. La photographie se situe à la fin du parcours du Mémorial alors que le visiteur vient de passer l'ensemble de l'exposition à prendre la mesure de l'horreur de la Seconde Guerre mondiale. Situer cette photographie au fond d'un bunker recomposé pour la circonstance, ne peut qu'interroger sur le type d'émotion qu'elle est censée provoquer. Cette forme de sacralisation du passé, d'iconisation, ferme la possibilité d'explication de ce même passé4. De plus, la mémoire de la Shoah semble recouvrir toute autre mémoire ; autre mémoire qui devient dès lors injustement inaudible.

De même, qui est le général Friedrich-Wilhelm Richter et quel peut être son lien avec Anne Frank ? Il n'est pas indiqué que ce général appartenait à la Waffen-SS, branche militaire des SS. Il est précisé dans la présentation de cet ensemble muséographique, que la photographie d'Anne Frank permet de se "rappeler" que la Shoah continua après le 6 juin $1944^{5}$. Cependant d'un point de vue scénographique et historiographique, le lieu où est située cette photographie pose de nouveau la question du rôle de l'armée allemande lors des crimes génocidaires nazis, sans y répondre. En mélangeant sciemment des récits complexes (le génocide des Juifs, les crimes de l'armée allemande contre ces mêmes Juifs, l'histoire militaire de la Seconde Guerre mondiale), le public n'est plus guidé par la connaissance mais uniquement par l'émotion. Il n'en tire aucun enseignement historique sinon la simple condamnation de l'horreur des faits par le "choc" des photographies... 


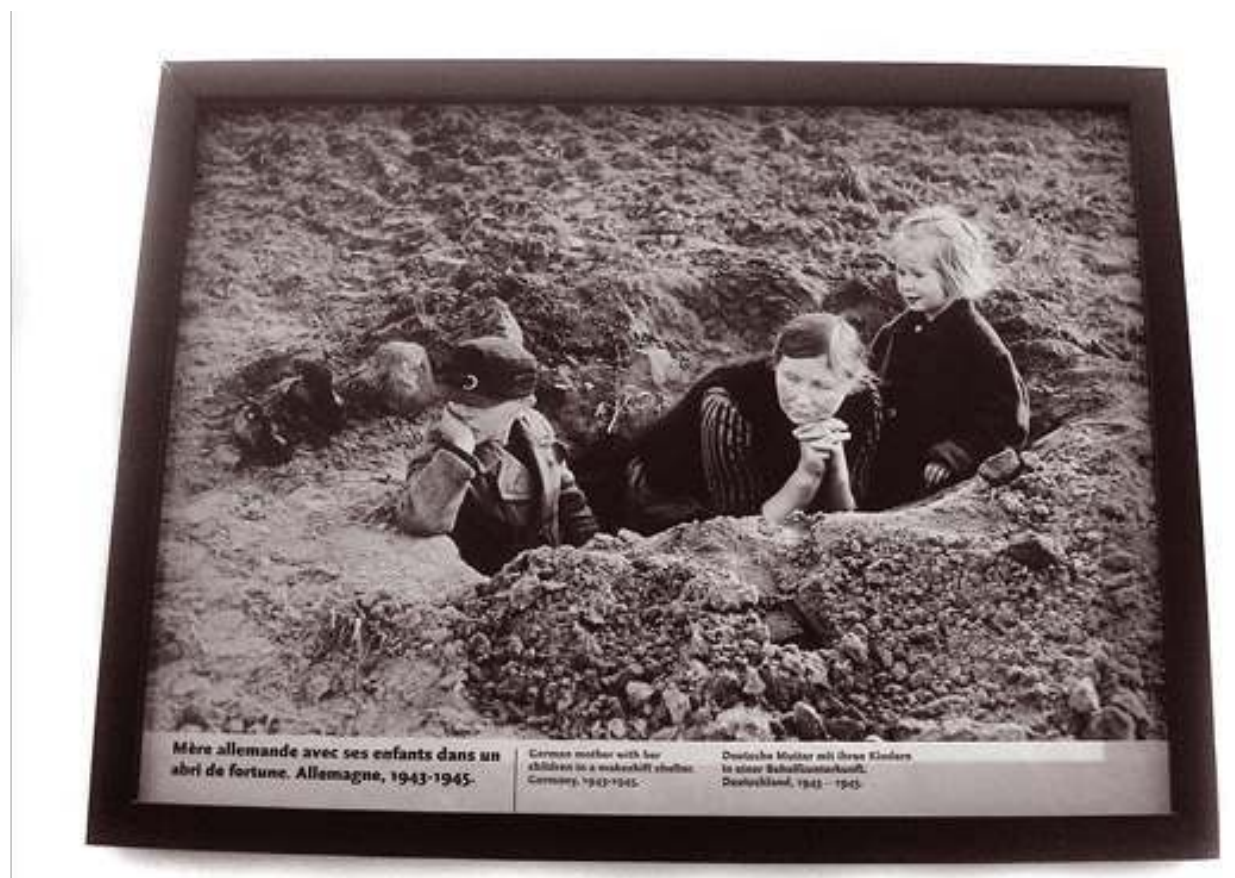

10 Cependant, ce "choc" peut-être salutaire lorsque, dans ce même Mémorial, sont exposées plusieurs photographies des victimes des bombardements alliés en Allemagne à Mannheim, Dresde ou à Munich par exemple ${ }^{6}$. Le Mémorial des civils dans la guerre à Falaise $^{7}$ expose aussi à partir d'une scénographie largement inspirée du Mémorial de Caen des civils allemands, victimes des bombardements en Allemagne. Mais ce sont surtout les victimes normandes que l'on découvre dans ce Mémorial. Quoiqu'il en soit, le statut de victimes face notamment à la violence des témoignages, mérite une médiation.

\section{Les armes allemandes exposées dans les sites et musées normands}

11 Pour améliorer les dispositifs d'accueil des publics, le Conseil régional de Normandie a préconisé d'améliorer la qualité de la médiation en ancrant les faits et le discours dans une vision contemporaine de l'histoire sur le territoire historique de la Bataille de Normandie ${ }^{8}$. En effet, un médiateur ou une médiatrice aurait tout intérêt à connaître les avancées historiographiques concernant la période de la Seconde Guerre mondiale. Il serait intéressant aussi, de mettre en avant l'histoire des sciences et des techniques de l'armement et de connaître les conséquences des armes et des bombardements sur les corps de manière très concrète. Car finalement, ce sont surtout des armes allemandes que l'on trouve dans ces sites et musées.

12 Un exemple, celui du Goliath, char robot téléguidé, qui était utilisé par la Wehrmacht lors de la Seconde Guerre mondiale. Il disposait d\&apos;une charge d\&apos;explosifs allant jusqu'à 90 kilogrammes de Cheddite (explosif très puissant). Chaque Goliath était prévu 
pour être détruit avec sa cible. Le 6 juin 1944, de jeunes soldats américains prennent pour un jouet sur la plage d'Utah Beach, ce char minuscule : ils sont déchiquetés'. Dans le musée d'Utah Beach, nous pouvons voir dans la première salle à gauche de l'entrée, une courte vidéo montrant le char Goliath en action. Au musée de la Libération, à Cherbourg, nous pouvons voir un char Goliath d'époque.

Au musée du débarquement Utah Beach : la guerre et les enfants quelle médiation utile?

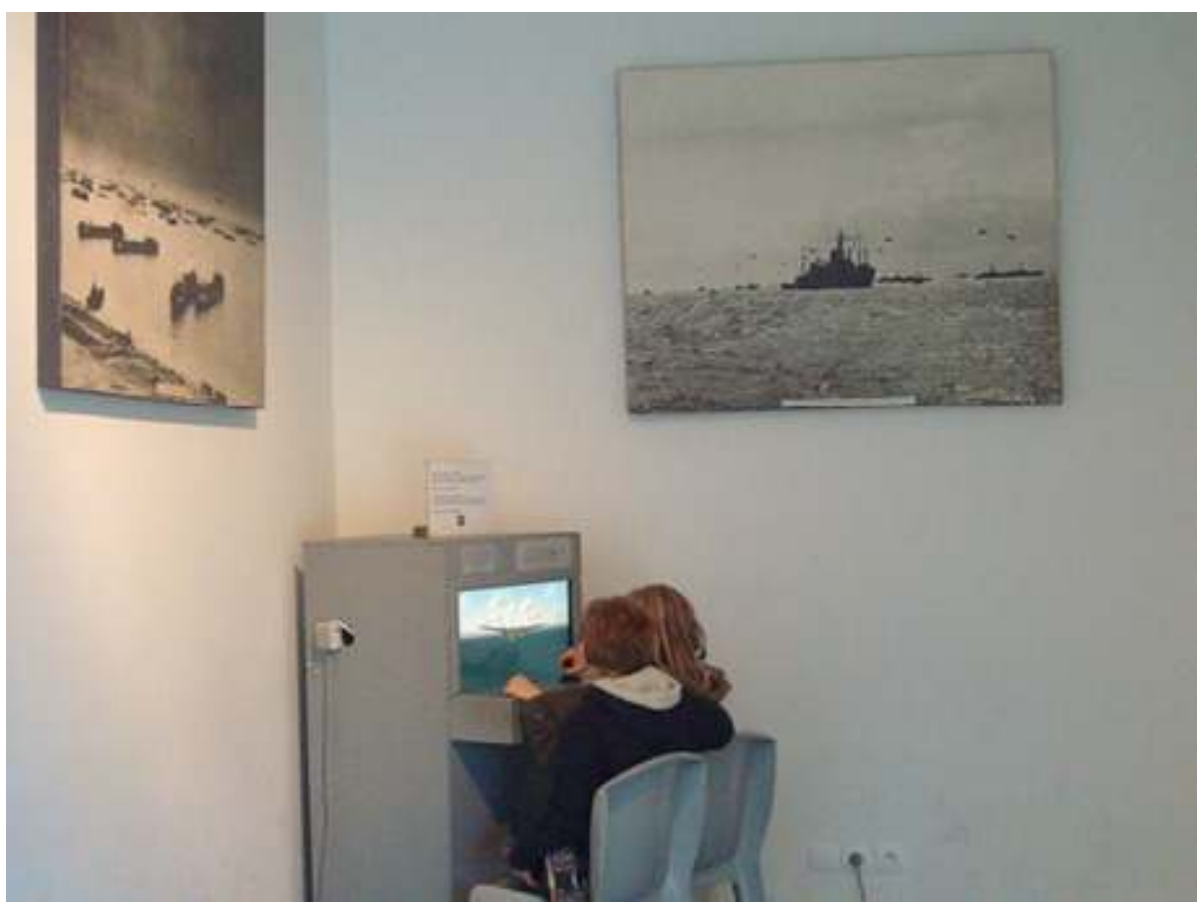

(c) Constance Micalef-Margain

Les musées et sites normands présentent à l'intérieur et à l'extérieur, une quantité impressionnante d'armes allemandes d'époque qui témoigne de la violence massive, industrielle de la Seconde Guerre mondiale. Et ceci, sans proposer non seulement une médiation, mais non plus d'explications des effets de ces armes sur les corps humains. Cet arsenal militaire exposé au regard d'enfants en bas âge mais aussi d'adolescents et de jeunes adultes pourrait être médié. Dans ce cadre, quelle pédagogie de la paix pourrait être mise en œuvre? Ces médiations pourraient avoir différents supports sachant que le numérique occupe aujourd'hui une place prépondérante dans les musées et les écoles.

La visite des musées en contexte scolaire permet de faire accéder directement les élèves à des informations sur le passé. Or, la mise en valeur d'expôts liés à la Seconde Guerre mondiale et le contexte historique de la fondation de ces sites muséaux qui les regroupent ne sont pas des faits neutres. Dès lors, comme le préconise l'universitaire Frédéric Rousseau (professeur d'histoire contemporaine à l'université de Montpellier), il faudrait que les visites scolaires dans les musées s'accompagnent de démarches de muséohistoire ${ }^{10}$ qui permettent aux publics scolaires en particulier de comprendre que la mémoire est une construction dont il faut savoir appréhender les ressorts. Ainsi, la narration des musées s'accompagnerait d'une approche critique de leur construction ou de leur scénographie. Ces propos compléteraient la narration muséale propre à chaque musée. Enfin, des ateliers de médiation qui interrogeraient des archives, des questionnaires plus classiques sur les lieux, les expôts ou encore des rencontres avec des 
œuvres d'artistes qui éclaireraient l'histoire en la confrontant à leurs œuvres par exemple, seraient des pistes didactiques pour que les musées d'histoire deviennent des lieux d'apprentissage ${ }^{11}$.

\section{Des scénographies particulières}

15 Au musée de la Libération à Cherbourg, l'exposition temporaire Regards croisés. Prisonniers ici et là-bas. Gefangene hier. Gefangene dort (8 mai-29 novembre 2015) plaçait dans un même essai de compréhension historique, le vécu des prisonniers allemands et français. Nous pouvions trouver ce mot d'une visiteuse dans le livre d'Or autour de l'exposition : "L'histoire écrite sous différents angles! Merci !"12. Dans cette exposition temporaire, était prônée une réconciliation entre histoire et mémoire par la création d'une scénographie nouvelle qui s'appuyait sur les avancées de la recherche scientifique.

Dans ce même musée, dans l'espace de l'exposition permanente, des photographies de la jeunesse française sous Vichy et de la jeunesse allemande sous Hitler sont présentées dans deux salles qui se font face dans le secteur "La Libération, montée vers la Lumière". Ce face à face est une belle illustration d'une histoire parallèle entre les deux pays. La scénographie différencie cet étage avec celui sombre du sous-sol qui correspond principalement aux années de l'Occupation. Ainsi, l'identification à la fois de l'éclairage (faible au sous-sol, éclairage du jour au rez-de-chaussée) et de l'architecture (les escaliers qui délimitent les deux espaces) sont des intentions muséales qui pourraient être décryptées par des élèves. Ces éléments scénographiques contrastés sont des moyens simples pour faire comprendre symboliquement la plongée dans le noir, le temps de la guerre, et le renouvellement de la démocratie par l'arrivée de la lumière.

Sites et musées inscrivent leurs histoires dans un temps mémorial précis. Restent aussi des traces d'un temps historiographique passé. Sur le site extérieur de Saint-Aubin-surMer, autour d'une batterie préservée, nous pouvons trouver gravé sur trois plaques en cuivre en anglais, allemand et français, un récit des événements. À leur lecture, nous nous rendons compte que si les textes anglais et allemand correspondent, ce n'est pas le cas du texte français auquel une phrase est rajoutée aux textes anglais et allemand : "(...) mais les quelques 70 douilles vides trouvées autour de l'emplacement témoignaient de la détermination avec laquelle ses servants s'étaient défendus". Si l'on peut difficilement remettre en cause la détermination des soldats allemands à se défendre, il faut s'interroger sur les destinataires de cette phrase uniquement en français. Il y a tout lieu de penser qu'elle s'adresse à des témoins ou même des acteurs français des événements, dans un dialogue entre histoire et mémoire. Or, ces acteurs et/ou témoins disparaissent peu à peu...

Le tourisme de mémoire implique une sauvegarde, une interprétation, une mise en valeur du patrimoine historique. Dans le cadre des sites et musées dédiés à la Seconde Guerre mondiale en Normandie, il doit présenter les vainqueurs, les vaincus et les civils dans la guerre. Exposer la guerre sur le lieu même des faits pousse à l'hypermnésie : un trop de mémoire organisé autour d'un seul espace-temps, la Normandie et le Débarquement et la Bataille de Normandie. Cette "mémoire saturée" empêche certains dialogues avec l'histoire. De plus, il n'existe pas de mémoire collective du Débarquement, même si celuici reste "le marqueur central de toutes les mémoires" ${ }^{13}$. Rajoutons que la mémoire est une représentation mentale du passé tandis que l'histoire met à distance les événements et permet donc une réflexion. Cette réflexion peut se fondre dans une médiation. 
Une mémoire qui dure... comme cette casemate à Saint-Aubin-sur-Mer avec un canon antichar allemand et sa plaque explicative.
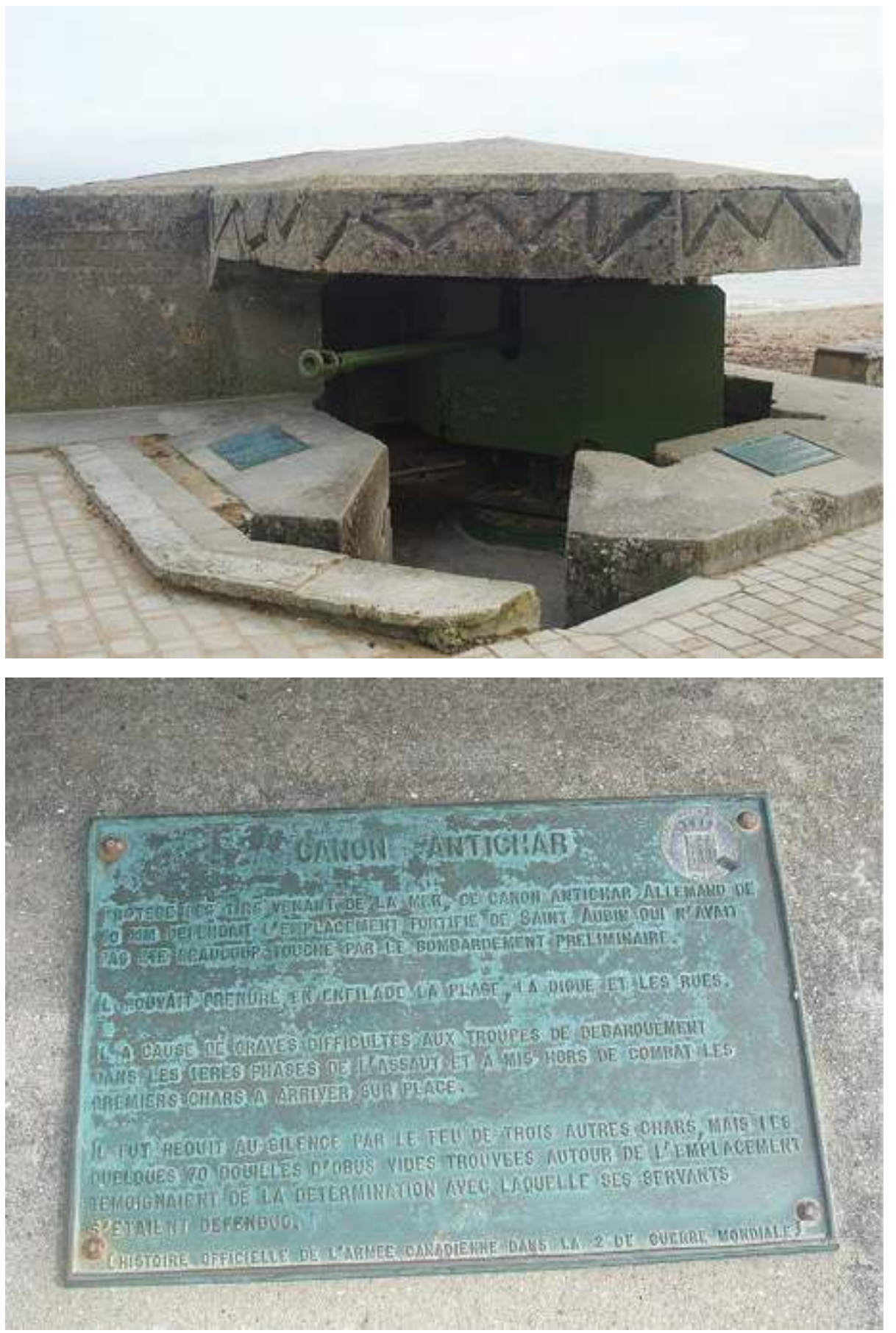

(c) Constance Micalef-Margain

Cette médiation a comme enjeu la construction de l'Union européenne, et tout le moins le prolongement de la pacification du territoire européen. Comme l'a souligné l'anthropologue anglaise Sharon MacDonald, l'Europe devenue un territoire mémoriel ("Memorylands"), institue dans chacun de ses musées, une mémoire mais aussi des valeurs. Les musées exposent l'héritage culturel du futur ${ }^{14}$. Dans cette optique, le passé qui y est exposé, est transcendé, choisi dans ses composantes, pour permettre une 
réflexion citoyenne sur une société à construire en commun ${ }^{15}$. Le musée est un instrument de pacification sociale, de digestion du passé, un lieu de réparation qui vise à la réconciliation des moments de discordance ${ }^{16}$.

Ainsi, ce tourisme de mémoire dans les musées de la Seconde Guerre mondiale en Normandie, oblige à organiser une médiation culturelle consciente des enjeux historiographiques contemporains et d'une nécessaire pédagogie de la paix. Ce travail permettrait la compréhension des événements dans une perspective éducative c'est-àdire en souhaitant que de tels événements (la guerre, les bombardements, la déportation, la violence, le racisme...) ne se renouvellent pas.

Si ces ateliers, ces médiations de pédagogie de la paix étaient mis en place, cela permettrait à l'évidence de remplir les objectifs les plus importants que s'est donné l'UNESCO jusqu'en 2021: valoriser l'éducation pour la paix, le dialogue culturel et la conciliation entre histoire et mémoire.

\section{BIBLIOGRAPHIE}

Becker, A. et Debary, O. (dir.) Montrer les violences extrêmes : théoriser, créer, historiciser, muséographier. Paris : Creaphiseditions, 2012.

Louvier, P., Mary, J. et Rousseau, F. (dir.) Pratiquer la muséohistoire. La guerre et l\&apos;histoire au musée. Pour une visite critique. Paris : Athéna Éditions, 2012.

Macdonald, S. Memorylands. Heritage and Identity in Europe Today. Oxford. New York : Routledge, 2013.

Macdonald, S. Difficult Heritage. Oxford. New York : Routledge, 2009.

Martens, S. (dir.) La France, 1\&apos;Allemagne et la Seconde Guerre mondiale : quelles mémoires ? Bordeaux : Presses universitaires de Bordeaux, 2007.

Peccatte, P. La fabrication de la renommée d'une ville : Sainte-Mère-Église. 2013 : http:// culturevisuelle.org/dejavu/1519

Wahnich, S. (dir.) Réfléchir l'histoire des guerres au musée, Culture et Musées, n²0, 2013.

Résistances au musée, enjeu, histoire et mémoires vivantes, Revue pluridisciplinaire de la fondation pour la mémoire de la déportation, n²1, juin 2013.

\section{NOTES}

1. www.airborne-museum.org/. Sur les prisonniers allemands dans ce même musée, voir : www.ouest-france.fr/normandie/manche/un-camp-de-prisonniers-allemandsfoucarville-4242153.

2. Chaumier, S. Traité d'expologie. Les écritures de l'exposition. Paris : La Documentation française, 2012.

3. www.utah-beach.com/ Visite effectuée à l'automne 2015. 
4. Mesnard, P. La question du pathos dans les espaces des musées et des mémoriaux, "Autrement dit, il s'agirait moins de parler de l'articulation entre émotion et raison explicative, que de considérer si cette articulation comporte ou non des procédés de mise à distance qui ont pour vocation de réguler la question de l'identification et de contenir ses dangers", in Becker, A. et Debary, O. (dir.) Montrer les violences extrêmes : théoriser, créer, historiciser, muséographier. Paris : Creaphiseditions. 2012, p. 77.

5. www.memorial-caen.fr/musee/exposition-permanente/debarquement-bataille-normandie/ bunker-souterrain

6. Sophie Wahnich a commenté ces photographies des bombardements des villes de Londres, Coventry, Hambourg, Dresde, Caen, Hiroshima, Leningrad au Mémorial de Caen, qui parce qu'elles n'étaient exposées "sans souci de hiérarchisation, de classement ou de contextualisation (...) rendaient ces bombardements équivalents". Ce qui permettait au "discours tenu par l'État français en 1944 sur les morts occasionnées par la Libération du territoire par les alliés [d'être] le même. Au nom du patrimoine détruit, de morts innocents, Laval disqualifiait les prétendus libérateurs". Wahnich, S. Les musées d\&apos;histoire du XXe siècle en Europe, Études, $n^{\circ} 7,2005$, pp. 29-41 .

7. www.memorial-falaise.fr/

8. Tourisme de mémoire en Normandie, synthèse établie par le service tourisme de la région Basse-Normandie, février 2014. Je remercie le personnel du Conseil régional de (Basse-) Normandie pour toute la documentation qui m'a été aimablement communiquée (services Tourisme, Documentation et Culture).

9. Carrel, P. Ils arrivent! Le Débarquement vécu du côté allemand. Paris : Robert Laffont, 2002 [1962], p. 77 : "Un groupe de 15 à 20 hommes [des soldats américains qui arrivent sur la plage d'Utah Beach, le 6 juin 1944] s'est arrêté à proximité d'un des minuscules "goliaths", échoué sur le sable. (...) [un] homme rampe jusqu'au joujou char, soulève le capot avant et, glissant à l'intérieur sa grenade qu'il amorce, fait un bon en arrière en riant de sa bonne farce. (...) Quelques secondes plus tard, leur groupe entier n'est plus, poumons arrachés, thorax crevés, qu'un amas de chairs pantelantes éparses sur le sable".

10. À l'école de Clio, dossier $n^{\circ} 1$. Récits et mises en texte du passé, 2015, http:// ecoleclio.hypotheses.org/135. Texte de Frédéric Rousseau.

11. Brusa, A. De la mauvaise et de la bonne didactique. Notes pour une pédagogie historique dans les musées de guerre, in Mary, J. et Rousseau, F. (dir.) Entre Histoires et Mémoires. La guerre au musée. Essais de muséohistoire. Paris : Michel Houdiard Éditeur, 2013, pp. 44-54.

12. Traduction de l'auteure : "Geschichte aus einem viel drehtenen Sicht. Merci !".

13. Laborie, P. La commémoration et ses trous de mémoire : le discours des notables locaux, in Rencontres internationales, Normandie (6 juin 1944) : l'émergence d'une mémoire collective? Actes du colloque du 15-17 juin 2011, Caen : Mémorial de Caen/Région Basse-Normandie, p. 125.

14. Macdonald, S. Memorylands. Heritage and Identity in Europe Today. Oxford. New York : Routledge, 2013 ; Macdonald, S. Difficult Heritage. Oxford. New York : Routledge, 2009.

15. Voir aussi les résultats du projet européen mené par Dominique Poulot (Université de Paris I, La Sorbonne), entre 2010 et 2013, intitulé EuNaMus pour European National Museums: Identity Politics, the Uses of the Past and the European Citizen. Ce projet de recherche a mis en lien les musées et leur rôle dans l'arène publique. Ainsi, les musées ne sont plus seulement des lieux d'exposition, de préservation, de conservation d'œuvres d'art... mais également des espaces de création de l'histoire et à ce titre ont un rôle à jouer auprès des citoyens.

16. Becker, A. et Debary, O. (dir.), op. cit., p. 16. 


\section{RÉSUMÉS}

Cette approche critique des musées du Débarquement et de la manière dont ils mettent en scène les combattants allemands plaide en faveur d'une médiation culturelle qui, dans une perspective éducative et dépassant l'aspect nécessairement émotionnel des lieux, prendrait en compte les enjeux historiographiques contemporains pour permettre une meilleure compréhension des événements et faire de ces musées de véritables outils de dialogue et de pacification des mémoires.

INDEX

Mots-clés : musée, guerre, normandie, mémoire

\section{AUTEUR}

\section{CONSTANCE MICALEF MARGAIN}

docteure en Histoire contemporaine

cmicalefmargain@gmail.com 